\title{
Cerebral palsy diplegia: improvements for walking
}

\section{Much publicity about Hungarian methods has ignored developments in Britain}

Ever since Little described cerebral palsy in $1862^{1}$ treatment has been given - by both rational and irrational methods - in the hope that physical improvement will occur and the child will be able to walk. In those with diplegia, though progress is slow and often tedious, the posture usually improves, balance matures, and walking may be possible by the age of 2 or 3 . Beyond this age different treatment methods are used, sometimes uncritically, by different professionals.

Such is the diversity of the condition of cerebral palsy and the treatment regimens used that acrimonious argument often occurs among centres offering treatment. This may cloud the issue of effectiveness of treatment. One of the most potent changes is the maturation that occurs with age and growth. Beals showed that the motor performance of spastic diplegic children changed up to the age of 7 and that improvements seen are not necessarily related to treatment. ${ }^{2}$

Last week the Health Minister, Mr David Mellor, told parliament that local authorities will be able in future to send children abroad for treatments not available in Britain. $\mathrm{He}$ gave the Petö Institute in Hungary as an example. Whether "conductive education" as given at the Petö Institute is better than other treatments has yet to be proved. On p 1145 Drs Robinson, McCarthy, and Little, all paediatricians, recount their experience of a visit to Hungary. The conclusions about the use of such a novel education method are of great importance to the future influence of this system on British care. Instead of being the media led "hype" and relaying the fascination that the Petö method seems to engender in the public in Britain the report simply states what was observed.

Clearly, patients with cerebral palsy are carefully selected at the Petö Institute, and those treated usually benefited from the intensive and coordinated work. The authors speculate on whether a "been to Hungary" label is worth while and conclude that treatment regimens in Britain are at best as good as those in the Petö Institute. It is unnecessary for all the children with cerebral palsy in Britain to rush abroad. Dr Robinson and colleagues compliment conductive education as a model for the multidisciplinary system of care we believe in and try to practise. They were also impressed by the use of all activities to improve function-not merely a short physiotherapy session in the school for the physically handicapped. It is thus all the more important that the unique Petö method is currently being tested in Britain by the Department of Education and Science in Birmingham.
"Conductors" are being employed to train children in an environment dedicated exclusively to the method.

Sadly, the surge of publicity for the Petö method has ignored entirely and unfairly the devoted work of therapists in British schools for the handicapped. Worse, even, is the general paucity of knowledge in Britain about other treatment methods, particularly, more exact surgery, perhaps because such methods have always seemed too "hit and miss."

Recently the fundamental divisions between operative and manipulative treatments have begun to change, and a timely re-evaluation is being made in the light of current advances. Physical maturation is slowed in children with cerebral palsy, ${ }^{2-4}$ but walking will eventually improve, especially in those with diplegia. This means that surgeons should encourage treatment by physiotherapy up to the age of 7 and reserve operative intervention until after that age. Such a policy should prevent parents and children believing that an operation "made the children worse." It often has done, and it is only recently that surgeons have realised that their intervention in the maturing, growing diplegic child can easily upset that maturation process. For the younger child manipulative treatment, physiotherapy, conductive education, and judicious use of splints, plaster, and stretching are likely to improve walking-either totally or enough to allow walking to be more than a hated physiotherapy progression over some metres in a gymnasium.

Surgeons and therapists must also acknowledge the part played by dynamics and biomechanics in walking, as the effects of surgery can be overwhelming. For example, surgeons have long been puzzled as to why a crouch gait develops after some procedures of lengthening the Achilles tendon but not after others. Similarly, a side or drastic abductor release may occasionally expose a child to the imbalance of spastic hip abductors, producing a very tiring and ugly, wide based hip abduction walk. The lack of reliable benefit from surgery has led to empirical or idiosyncratic treatment regimens that require surgery every year and physiotherapy afterwards for up to a year until more surgery is undertaken - the so-called "birthday syndrome."

The way forward is, we believe, to build on the improvements provided by logical investigation and then treatment. This change has been led by orthopaedic surgeons in North America and has produced some spectacular cosmetic advantages for some patients with spastic diplegia." A 
secondary gain may be some lowering in the energy cost of the walk so that the patient is less tired, and this has obvious implications for long term walking capability.

A successful outcome in medicine requires that diagnosis should precede treatment. For the diplegic patient we need to improve our diagnostic capabilities by studying the moving child. The potential for improved walking may be predicted by an evaluation in a gait laboratory when the child's physical maturation is coming to a plateau at about 7 years. Many advanced gait laboratories exist and are mostly dedicated to research. What patients need are more clinically based laboratories that can make kinematic recordings formally, the video recordings obtained being used to help clinical decision making. Instrumentation may also be used to assess the degree of handicap, and multiple tests may be applied - for example, estimates of the energy cost of walking, dynamic electromyography recordings for establishment of muscle contraction patterns, and even production of vector force diagrams around the leg joints. Such scientific analysis allows guess work and intuition to give way to understanding of particular sequences of movements. Similarly, a telemetry signal of heart rate can be used to estimate the "physiological cost" of walking when the resting rate is subtracted from the exercising rate and then divided by the speed obtained during that walk.

Logical suggestions for treatment may be made when these details are provided for the clinician. For example, a dynamic flexion deformity of the knee or hip may be preventing a correct step forward in the progression line. The patient's history (especially of mobility problems) and the known static deformities discovered at the initial physical examination may be combined with a study of the kinematic and kinetic data to suggest a logical programme of surgical or other treatment.

Such analysis of the walking pattern in the presence of the child's own physiotherapist and parent will often produce suggestions that may then be tested in the clinical gait laboratory - for example, in altering the footwear or providing an ankle foot orthosis (splint) - even if operative treatment is not indicated.

Surgical thinking and procedures have also changed. The "birthday syndrome" is the reminder of sometimes inappropriate surgery being applied to a patient in a haphazard way. ${ }^{5}$ The pioneering work of Gage and Sutherland in the United States has identified surgical treatment regimens that are understandable in the context of the pathological and clinical effects of diplegia. ${ }^{67}$

A thorough assessment of gait may show that it is being obstructed by restrictions of joint range. For example, the rectus femoris muscle often prevents knee flexion in swing phase, preventing anything but a walk that has a high energy cost and is stiff kneed and spastic. In such cases a simple transfer of the tendon of this muscle may "unlock" the knee and lead not only to a huge cosmetic gain but also to a reduction in the effort of clearing the swing leg.

Other leg deformities may be dealt with in one session. Even so, bony procedures may need to precede the soft tissue releases by a few weeks so that mobilisation may begin within a few days of surgery. The hope is that a single event in hospital will lead to a clearer path to more "normal" walking. Certainly the cosmetic result is often very pleasing. ${ }^{68}$

The most important long term benefit seems likely to be a reduction in the physiological cost of walking. Preliminary studies in the clinical gait laboratory in Oswestry have confirmed this effect after 12 months in all 12 patients who were treated. This holds out the promise of continuing walking for many children with diplegia as they mature beyond childhood and adolescence.

Eliminating the spastic walk is impossible because the primary lesions cannot be altered. Debate about the treatment of the secondary effects is likely to continue, but we believe that with better diagnostic methods and more appropriate surgery performed at a correct age the end results should show some improvement in the next decade.

Director,

JOHN PATRICK

Orthotic Research and Locomotor Assessment Unit,

Robert Jones and Agnes Hunt Orthopaedic Hospital,

Oswestry, Shropshire SY10 7AG

1 Little WJ. On the influence of abnormal parturition, difficult labours, premature birth, and asphyxia neonatorum, on the mental and physical condition of the child, especially in relation to deformities. Transactions of the Obstetrical Society of Londom 1862;3:293. (Reprinted in Cerebral Palsy Bulletin 1958;1:5-36.

Beals RK. Spastic paraplegia and diplegia: an evaluation of non-surgical factors influencing the prognosis for ambulation. F Bone foint Surg [Am] 1966;48:827-46.

3 Sutherland DH, Olshen R, Cooper L, Woo SK-Y. The development of mature gait. F Bone foin Surg $[\mathrm{Am}]$ 1980;62:336-53.

Bleck EE. Locomotor prognosis in cerebral palsy. Dev Med Child Neurol 1975;17:18-25.

5 Rang M, Silver R, de la Garza J. Cerebral palsy. In: Lovell WW, Winter RB, eds. Pediatri orthopedics. 2nd ed. Vol 1. Philadelphia: Lippincott, 1986:345-96.

Gage J, Fabian D, Hicks R, Tashman S. Pre and post-operative gait analysis in patients with spastic diplegia: a preliminary report. I Pediatr Orthop 1984:4:715-25.

7 Sutherland DH, Larsen LJ, Mann R. Rectus femoris release in selected patients with cerebral palsy: preliminary report. Dev Med Child Neurol 1975;17:26-34.

8 Gage JR. Gait analysis for decision making in cerebral palsy. Bull Hosp ft Dis Orthop Inst 1983;43:147-63.

\section{Constipation in childhood}

\section{Treatment should be given early}

Most doctors who care for children will at some stage confront one with chronic constipation. What promises in its early stage to be a condition easily remedied by increasing the fluid and fibre intake gradually develops into a nightmare for all concerned. A mixture of painful defaecation, deliberate withholding of faeces, overflow faecal incontinence, and parental anxiety - all compounded by a physiological tendency to constipation - leads all too often to an ever increasing use of laxatives and diminishing confidence in the doctor.

Theories from child psychiatrists, ${ }^{12}$ paediatric surgeons, ${ }^{3}$ and paediatricians ${ }^{+6}$ on the aetiology of chronic constipation have been as varied as the many factors operating in each individual child. Loening-Baucke recently tried to determine which features might predict a good response to treatment.
Her regimen included the essentials: effective evacuation of retained faeces, maintenance of an empty rectum with oral or rectal medication, strict attention to diet and toilet routines, and a careful explanation of the pathophysiology. As might be expected, she found that those children who had presented with frequent overflow soiling and with faecal masses palpable on abdominal examination were less likely to be free of constipation and off laxatives after one year.

What is the place of specialist investigations? Anorectal manometry has been recommended for severly constipated children to help exclude ultrashort segment Hirschsprung's disease. ${ }^{5}$ There seems to be less inhibition of the internal sphincter muscle in response to rectal distension even in 